\title{
Alterações oculares em pacientes com tuberculose
}

\author{
Ocular manifestation in patients with tuberculosis
}

\author{
Simone Ribeiro Araújo de Almeida ${ }^{1}$ \\ Luciana Peixoto Finamor ${ }^{2}$ \\ Cristina Muccioli ${ }^{3}$
}

\section{RESUMO}

Objetivo: Analisar os achados clínicos da tuberculose ocular e seus aspectos epidemiológicos. Métodos: Foram revisados prontuários médicos de pacientes que foram atendidos no Departamento de Oftalmologia - setor de Uveíte e AIDS da UNIFESP-EPM, São Paulo, Brasil, entre janeiro de 1999 e julho de 2002 e que tiveram diagnóstico de tuberculose ocular. Foram inclusos os pacientes que apresentaram PPD forte reator, epidemiologia positiva para tuberculose e lesão ocular que melhorou com tratamento específico. Resultados: Foram analisados prontuários médicos de 17 pacientes, sendo $12(70,6 \%)$ do sexo feminino. A idade média foi de 54 (24-84) anos. O tempo médio entre o início dos sintomas e a primeira consulta foi de 100 dias. Os achados oculares foram inespecíficos nesta ocasião mas prevaleceram as alterações de segmento anterior e $41 \%$ dos pacientes apresentavam sinais de tuberculose sistêmica. Alterações como glaucoma, descolamento de retina e hemorragia vítrea foram responsáveis pela redução da acuidade visual. Conclusão: Embora pouco prevalente, a tuberculose ocular apresenta morbidade elevada. A inespecificidade da apresentação clínica da doença ocular pode atrasar o seu diagnóstico.

Descritores: Tuberculose ocular/complicações; Uveíte/etiologia; Glaucoma/etiologia; Descolamento retiniano/etiologia; Manifestações oculares

\section{INTRODUÇÃOO}

No Brasil há registro de 110.000 a 120.000 casos novos/ano de tuberculose sendo que a região norte apresenta a maior prevalência nacional ${ }^{(1)}$.

São fatores de risco para tuberculose: raça (não branca), sexo (masculino), idade (crianças e idosos), nível socioeconômico (baixo), doenças sistêmicas (diabetes melitus, tumores, alcoolismo, AIDS, silicose) ${ }^{(2)}$.

A tuberculose ocular é encontrada em 1 a $2 \%$ dos pacientes com doença pulmonar $^{(1,3)}$ e pode comprometer todas as estruturas oculares e seus anexos por ação direta da agente que, a partir de infecção inalatória e posterior disseminação linfo-hematogênica, acometem órgãos extrapulmonares ou por reação imunológica de hipersensibilidade do tipo IV.

As alterações mais freqüentemente causadas por ação direta do patógeno são o tuberculoma de anexos, conjuntiva, íris e coróide; as uveítes anteriores e posteriores; e a endoftalmite. A presença de flictênulas na conjuntiva ou córnea e de vasculite retiniana ocorrem devido à reação de hipersensibilidade tipo IV.

Devido a dificuldade em se isolar o agente na tuberculose ocular o diagnóstico é presuntivo na maioria dos casos, considerando para tanto a epidemiologia positiva, a clínica (sistêmica e ocular) e a resposta imunológica do paciente.

Este estudo foi realizado com o objetivo de estudar as alterações ocula- 
res em pacientes com diagnóstico provável de tuberculose ocular que foram avaliados no ambulatório do setor de Uveíte e AIDS do departamento de Oftalmologia da Unifesp-EPM no período de janeiro de 1999 a julho de 2002.

\section{MÉTODOS}

Em estudo retrospectivo, aprovado pela CEP-Unifesp, foram revisados prontuários médicos de 17 pacientes com diagnóstico provável de tuberculose ocular atendidos no setor de Uveíte e AIDS do departamento de Oftalmologia de UnifespEPM no período de janeiro de 1999 a julho de 2002. Foram avaliados os dados epidemiológicos, clínicos e de diagnóstico de cada paciente.

Considerou-se diagnóstico provável de tuberculose ocular casos de:

1. Epidemiologia positiva para tuberculose

2. $P P D \geq 10 \mathrm{~mm}$ (forte reator)

3. Alterações oculares em que não se identificou outro agente etiológico e que melhoraram com tratamento específico para tuberculose.

Todos os pacientes foram avaliados por um clínico geral ou infectologista para investigar focos de tuberculose extra-oculares.

Foram excluídos do estudo os pacientes portadores da síndrome da imunodeficiência adquirida (AIDS).

\section{RESULTADOS}

Do total de 17 pacientes, $12(70,6 \%)$ eram do sexo feminino e $5(29,4 \%)$ do sexo masculino; a idade média foi 54 anos (variando de 24 a 84 anos) e $35 \%$ eram brancos.

O teste de Mantoux (PPD) variou de 12 a $30 \mathrm{~mm}$ (média: $18,9 \mathrm{~mm})$.

As queixas oculares na ocasião da primeira consulta estão descritas na tabela 1. Quarenta e um por cento dos pacientes apresentaram alterações pulmonares compatíveis com tuberculose (Tabela 2). Não tivemos acesso aos exames realizados pelo clínico geral ou infectologista que apenas nos informou do comprometimento pulmonar sem especificar o tipo de alteração.

O tempo médio entre a primeira consulta oftalmológica e o diagnóstico ocular foi de 100 dias (13-427 dias) sendo que 6 pacientes $(35,3 \%)$ tiveram seu diagnóstico feito com tempo acima da média que foi de 100 dias.

\begin{tabular}{|lc|}
\hline \multicolumn{2}{|c|}{ Tabela 1. Queixas oculares em consulta inicial } \\
Queixa & Incidência (\%) \\
BAV súbita & 47,0 \\
Hiperemia & 47,0 \\
Embaçamento visual & 41,1 \\
BAV progressiva & 35,3 \\
Dor ocular & 35,3 \\
Fotofobia & 35,3 \\
Lacrimejamento & 29,4 \\
\hline
\end{tabular}

Em consulta inicial observou-se multiplicidade de alterações oculares (Tabela 3). A forma mais freqüente foi a panuveíte $(64,7 \%)$ bilateral (53\%) granulomatosa (52,9\%).

As principais complicações encontradas foram: aumento da pressão intra-ocular (PIO) $11,7 \%$; descolamento de retina $11,7 \%$; e hemorragia vítrea $11,7 \%$; edema macular $5,9 \%$ e catarata $5,9 \%$. Um paciente apresentou-se com quadro de esclerite nodular unilateral.

Os 17 pacientes avaliados neste estudo receberam tratamento sistêmico específico com pirazinamida, rifampicina e isoniazida pelo período de 6 meses.

Todos os pacientes apresentaram remissão da doença ocular e pulmonar após tratamento prescrito e a acuidade visual final melhorou em $29,4 \%$, manteve-se inalterada em $47,1 \%$ e piorou em 23,5\% dos pacientes. Dos 4 pacientes que apresentaram piora da acuidade visual, $3(75 \%)$ foram diagnosticados com mais de 100 dias após a primeira consulta (Tabela 4).

\begin{tabular}{|lc|}
\hline \multicolumn{2}{|c|}{ Tabela 2. Tuberculose associada } \\
Doença sistêmica & Incidência (\%) \\
Presente & 41,1 \\
Ausente & 58,9 \\
\hline
\end{tabular}

\begin{tabular}{|lc|}
\hline \multicolumn{2}{|c|}{ Tabela 3. Alterações oculares em consulta inicial } \\
Alterações oculares & Incidência (\%) \\
Patologia externa ocular & 5,9 \\
Esclerite & \\
Pressão intra-ocular & 11,8 \\
Glaucoma & \\
Segmento anterior & 35,3 \\
Catarata & 35,3 \\
Celularidade (CA) & 35,3 \\
"Flare" & 35,3 \\
Pks "Mutton fat" & 11,8 \\
Finos & 11,8 \\
Sinéquia posterior & 5,9 \\
Edema de córnea & \\
Segmento posterior & 35,3 \\
Coroidite & 11,8 \\
Hemorragia vítrea & 11,8 \\
Retinite exsudativa & 11,8 \\
Vasculite & 11,8 \\
Descolamento de retina & 5,9 \\
Vitreíte & 5,9 \\
Edema macular & \\
\hline
\end{tabular}

\begin{tabular}{|c|c|c|c|}
\hline $\begin{array}{l}\text { Tempo } \mathrm{p} / \mathrm{dx} \\
\text { Evol. AV }\end{array}$ & $\leq 100$ dias & $>100$ dias & $\begin{array}{c}\text { Total } \\
(100 \%)\end{array}$ \\
\hline Melhorou & $3(75,0 \%)$ & $1(25,0 \%)$ & 4 \\
\hline Inalterada & 7 (88,8\%) & $2(11,2 \%)$ & 9 \\
\hline Piorou & $1(25,0 \%)$ & $3(75,0 \%)$ & 4 \\
\hline
\end{tabular}




\section{DISCUSS $\tilde{A} O$}

Alguns autores afirmam que apesar da tuberculose ser a doença sistêmica de maior morbidade e mortalidade em países em desenvolvimento a incidência da doença ocular é baixa ${ }^{(4)}$. Em nosso serviço a tuberculose representou $1 \%$ dos casos de uveíte no período estudado. Também encontraram índices semelhantes em estudos epidemiológicos realizados no Hospital São Geraldo - MG $(0,9 \%)^{(5)}$.

Diferente do achado na literatura, a maior incidência de tuberculose ocular ocorreu em mulheres. Os aspectos idade e raça corroboraram com outros estudos ${ }^{(1,3)}$.

Em 41,1\% dos pacientes foi identificada tuberculose pulmonar em exame clínico. Não foi encontrada outra forma de doença sistêmica.

A característica endêmica da tuberculose em nosso meio, associada à vacinação infantil da BCG torna o teste positivo fraco reator, na maioria dos indivíduos, sem significar infecção pelo bacilo da tuberculose. Estudo realizado previamente mostrou que todos os indivíduos vacinados pela BCG com PPD > 22 mm estavam infectados com M. tuberculosis ${ }^{(6)}$.

É importante notar que este resultado não permite diagnosticar a tuberculose doença, apenas sugere infecção pelo patógeno, contudo, sua relevância está no fato de se apresentar reator em $21,4 \%$ dos pacientes com doença ocular e forte reator em $57,2 \%$ deles.

Assim como em outros estudos a forma mais comum da tuberculose ocular encontrada foi a uveíte crônica bilateral granulomatosa $(35,3 \%)$ ocorrendo contudo uma diversidade de apresentação clínica. As principais complicações foram a catarata $(35,3 \%)$, o glaucoma $(11,8 \%)$, a hemorragia vítrea $(11,8 \%)$, o descolamento de retina $(11,8 \%)$ e o edema macular cistóide $(5,9 \%)$, sendo que estas três últimas foram responsáveis por diminuição permanente da acuidade visual.

As queixas que motivaram os pacientes a buscarem atendimento oftalmológico foram bastante inespecíficas e presentes na maioria das uveítes. Os achados oculares foram também comuns a uveítes de variada etiologia. Por este fato a hipótese de tuberculose ocular é muitas vezes negligenciada havendo longo período entre a consulta inicial e o diagnóstico. Este estudo mostrou tempo médio de pouco mais de 3 meses (100 dias) chegando até a 1 ano e 2 meses (427 dias), período muito longo em nosso entender, que revela a dificuldade em realizar diagnóstico em uveítes posteriores.

É notável a importância da precocidade do diagnóstico quando observamos que $84 \%$ dos pacientes que apresentam melhora ou manutenção da acuidade visual tiveram intervalo menor ou igual a 100 dias entre a primeira consulta e o diagnóstico e que $75 \%$ daqueles que pioraram a acuidade visual tiveram intervalo maior de 100 dias.

Visto que a tuberculose ocular é uma doença crônica pleomórfica, com alto índice de redução da acuidade visual $(23,5 \%)$ e que o tratamento precoce aumenta a chance de manutenção ou melhora desta visão ${ }^{(6)}$, torna-se imperativo que a pesquisa desta etiologia seja realizada em todos os pacientes com uveítes crônicas de causa indeterminada.

\section{CONCLUSÃO}

Apesar de pouco prevalente, a tuberculose ocular apresenta morbidade elevada, podendo evoluir com comprometimento visual permanente em alguns casos. As queixas oculares iniciais e os achados clínicos foram inespecíficos para a realização do diagnóstico, havendo a necessidade de efetuar exames complementares que nem sempre são suficientes para definí-lo.

Devido a sua forma pleomórfica de apresentação clínica houve longo intervalo entre o início da queixa oftalmológica e o diagnóstico e apesar do pequeno número de pacientes, este estudo sugere que quanto mais precoce o diagnóstico for realizado, melhor o prognóstico visual.

\section{ABSTRACT}

Purpose: To analyze clinical findings of ocular tuberculosis and its epidemiological features. Methods: Were reviewed clinical files of patients who attended the Ophthalmology Uveitis and AIDS sector of UNIFESP-EPM, São Paulo, Brazil between January 1999 and July 2002 and had a diagnosis of ocular tuberculosis. Patients who had Mantoux test higher than $10 \mathrm{~mm}$, epidemiological history of tuberculosis and ocular findings that improved after specific treatment were included. Results: Seventeen medical files were analyzed, of those, $12(70,6 \%)$ were females. The mean age was 54 (24-84) years. The average time between symptoms and diagnosis was 100 days. Ocular findings at the first interview were multiple but most were at the anterior segment of the eye and $41 \%$ of patients who had clinical findings that suggested tuberculosis other than ocular. Situations as glaucoma, retinal detachment and vitreous hemorrhage were responsible for reduction of visual acuity. Conclusions: Ocular tuberculosis showed a low prevalence but important visual loosing morbidity. The multiplicity of clinical findings is responsible for the delay of diagnosis.

Keywords: Tuberculosis, ocular/complications; Uveitis/etiology; Glaucoma/etiology; Retinal detachment/etiology; Eye manifestations

\section{REFERÊNCIAS}

1. Oréfice F, Campos WR, Fernandes LC, Azevedo JF. Tuberculose. In: Orefice F. Uveíte, clínica e cirúrgica. São Paulo: Cultura Médica; 2000. p.431-41.

2. Helm CJ, Holland GN. Ocular tuberculosis. Surv Ophthalmol. 1993;38(3): 229-56.

3. Rosen PH, Spalton DJ, Graham EM. Intraocular tuberculosis. Eye. 1990;4(Pt 3):486-92.

4. Biswas J, Madhavan HN, Gopal L, Badrinath SS. Intraocular tuberculosis. Clinicalpathologic study of five cases. Retina. 1995;15(6):461-8.

5. Fernandes LC, Oréfice F. Aspectos clínicos e epidemiológicos das uveítes, em serviços de referência em Belo Horizonte, de 1970 a 1993. Rev Bras Oftalmol. 1996;55(8):569-92.

6. Prado WT, Rocha MOC, Pedroso RD, Fonseca JGM, Silva OA. Tuberculose: terapêutica clínica. Rio de Janeiro: Guanabara Koogan; 1998. 